\title{
Typhon: Multiplexed TEM Sample Preparation
}

\author{
Sean Mulligan ${ }^{1}$, Tilak Jain ${ }^{1}$, Erika Duggan ${ }^{2}$, Er Liu ${ }^{2}$, Jeffrey A. Speir ${ }^{1}$, Anchi Cheng ${ }^{1}$ John Nolan $^{2}$, \\ Bridget Carragher ${ }^{1}$ and Clinton S. Potter ${ }^{1}$ \\ 1. National Resource for Automated Molecular Microscopy, The Scripps Research Institute, La Jolla, \\ CA, USA \\ 2. La Jolla Bioengineering Institute, San Diego, CA, USA
}

Over the last decade there has been significant progress in the synthesis of inorganic and hybrid inorganic-organic nanoparticles in solution. Optimizing the synthesis conditions is often dependent on structural characterization of the nanoparticles. Transmission Electron Microscopy (TEM) provides a method for structural characterization that provides both high-resolution details of individual particles as well as particle size distribution and morphology. The draw back to this method has been that specimen preparation is typically very low-throughput; single specimens are prepared on individual TEM grids and then imaged one at a time. Imaging several tens to hundreds of nanoparticle synthesis conditions thus requires an equal number of TEM grids, which is cumbersome, time consuming, and not feasible as a routine characterization method.

We have developed a system that uses a low volume liquid handling system to place up to 96 individual samples onto a single TEM grid (figure 1). The system has been tested with gold nanorod samples that have been prepared by the seedless growth technique of sodium borohydride-mediated AuCl4 reduction in the presence of CTAB and AgNo3. Reactant concentrations were varied to find the conditions that yielded the smallest and most uniform Au nanorods. A sciFLEXARRAYER S3 system (Scienion US Inc.) was used to transfer 96 nanorod samples from a well plate (Grenier Bio-One) onto a $50 \mathrm{~nm}$ thick, 1

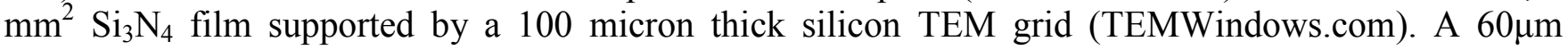
nozzle tip with a hydrophobic coating was used to dispense $240 \mathrm{pL}$ drops at a spacing of $\sim 100 \mu \mathrm{m}$. The total preparation time for two duplicate 96 sample TEM grids was 380 minutes.

The Leginon software system [1] was used to obtain TEM images of all samples on the grid at multiple scales, starting with an overall atlas of the entire grid. Intermediate magnification images are then used to target within each spot and a total of 10-20 pairs of high magnification images $(25,000 \mathrm{x}$ and $62,000 \mathrm{x})$ are acquired for each sample spot. Total imaging time is approximately $6 \mathrm{hrs}$ for all 96 samples.

We have demonstrated the feasibility of using a low volume liquid handling system to place up to 96 individual samples onto a single TEM grid (figure 2). The sample spots are regularly spaced and the spot diameter varies from $\sim 15$ to $\sim 30 \mu \mathrm{m}$. The particle distribution within the spot and the diameter of the spot are dependent on the buffer composition and grid substrate surface treatments. We are continuing to optimize both the grid preparation as well as the automated TEM data collection strategies and expect that the system will significantly increase the throughput and lower the cost for using TEM as a routine characterization method for nanoparticle synthesis.

\section{References:}

[1] C. Suloway et al, J. Struct. Biol 151 (2005) p. 41-60

[2] The authors acknowledge funding from the National Institutes of Health (GM103966, GM103310, and EB003824). 


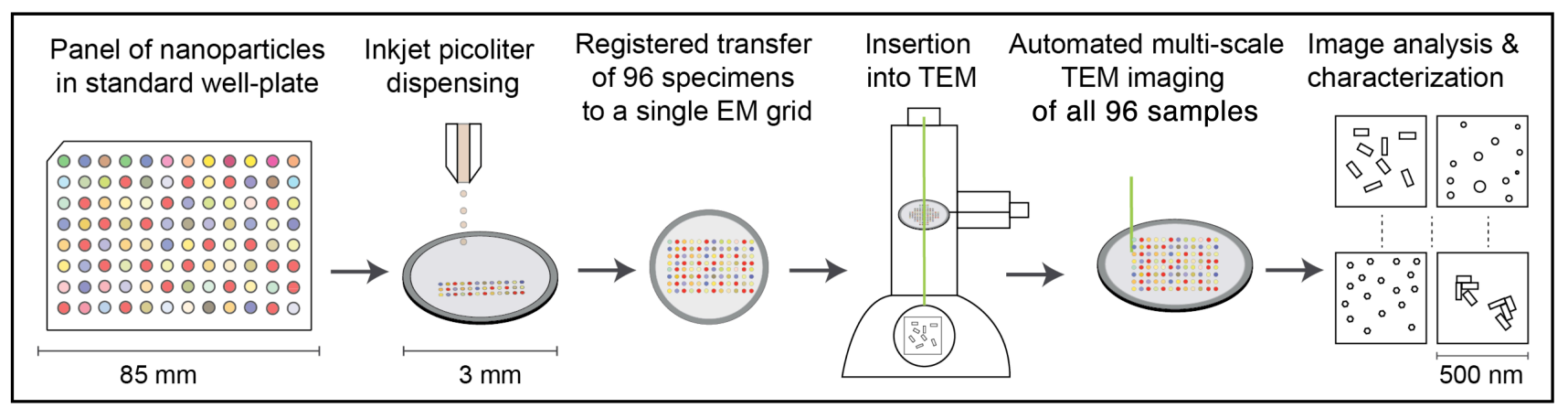

Figure 1. High-Throughput TEM Specimen Preparation Using Piezo-Based Picoliter Dispensing Inkjet transfer of multiple samples from well-plates to single EM grids followed by automated multi-scale TEM imaging. The goal is to increase the throughput of the imaging by $100 \mathrm{x}$ and reduce the sample volumes by $1000 x$.

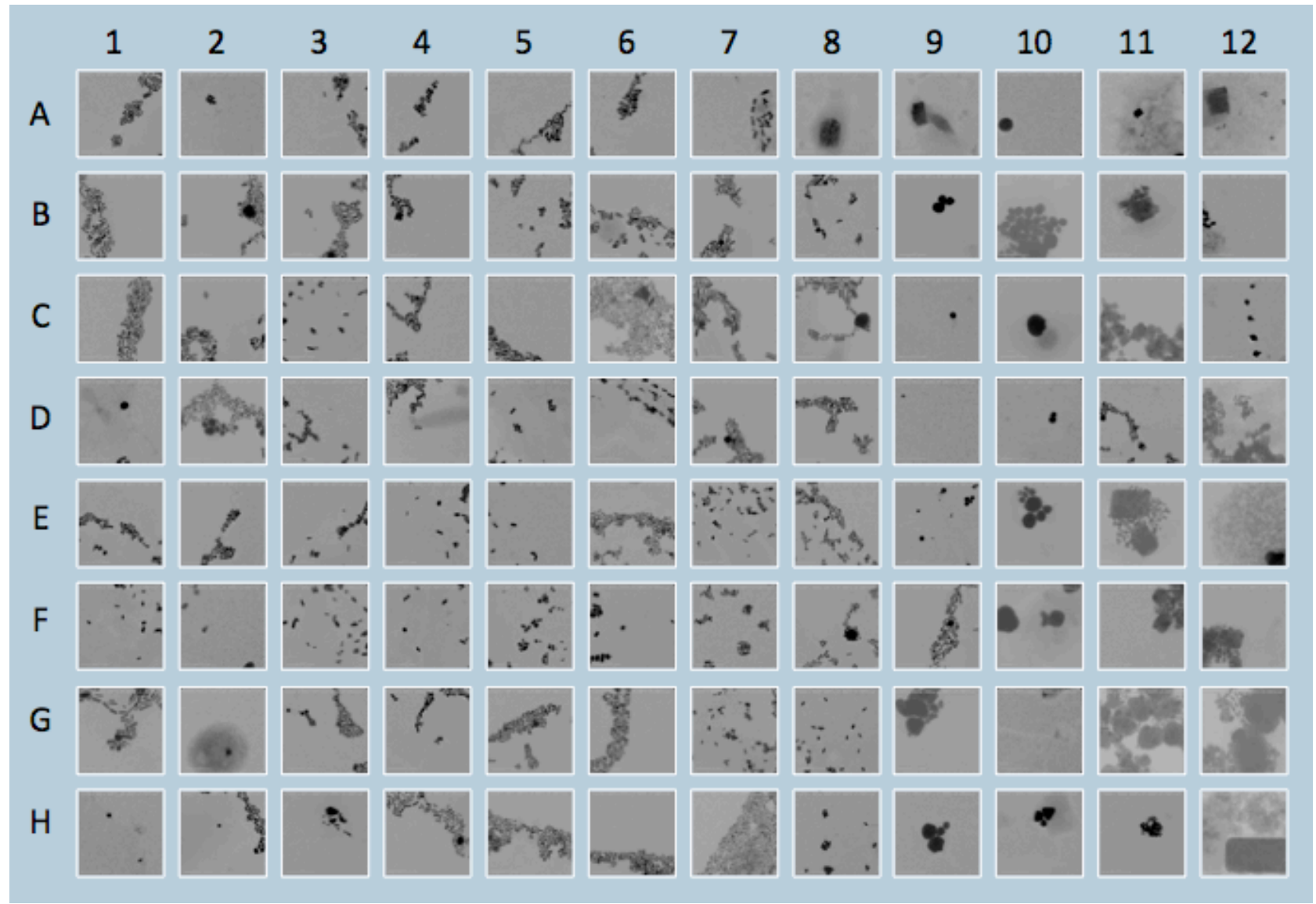

Figure 2. Thumbnail images (62,000x magnification) of 96 different samples from a single TEM grid; 10-20 images of each sample were obtained, which is sufficient to provide a statistically useful sample size. 